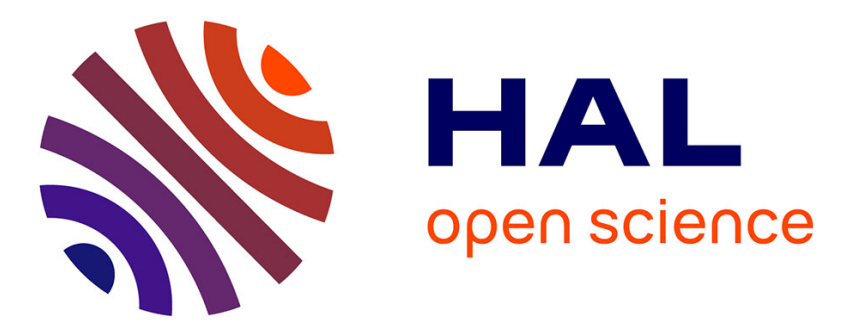

\title{
RAIVE (RhumAtologie InterVentionnelle et Échographie) : résultats d'une enquête nationale
}

\author{
Aurélie Sicaud
}

\section{To cite this version:}

Aurélie Sicaud. RAIVE (RhumAtologie InterVentionnelle et Échographie) : résultats d'une enquête nationale. Médecine humaine et pathologie. 2012. dumas-00746775

\section{HAL Id: dumas-00746775 https://dumas.ccsd.cnrs.fr/dumas-00746775}

Submitted on 29 Oct 2012

HAL is a multi-disciplinary open access archive for the deposit and dissemination of scientific research documents, whether they are published or not. The documents may come from teaching and research institutions in France or abroad, or from public or private research centers.
L'archive ouverte pluridisciplinaire HAL, est destinée au dépôt et à la diffusion de documents scientifiques de niveau recherche, publiés ou non, émanant des établissements d'enseignement et de recherche français ou étrangers, des laboratoires publics ou privés. 


\section{Université \\ Joseph Fourier \\ MEDECINE}

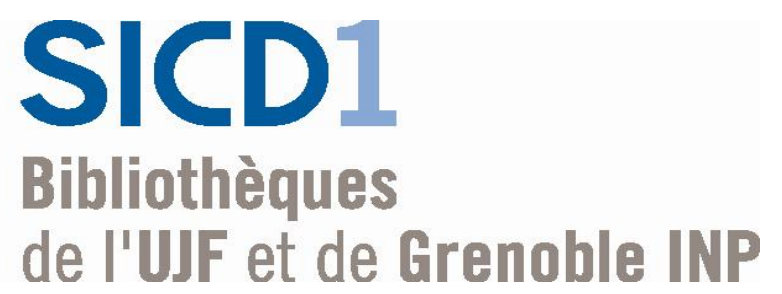

\section{AVERTISSEMENT}

Ce document est le fruit d'un long travail approuvé par le jury de soutenance et mis à disposition de l'ensemble de la communauté universitaire élargie.

Il n'a pas été réévalué depuis la date de soutenance.

Il est soumis à la propriété intellectuelle de l'auteur. Ceci implique une obligation de citation et de référencement lors de l'utilisation de ce document.

D'autre part, toute contrefaçon, plagiat, reproduction illicite encourt une poursuite pénale.

Contact au SICD1 de Grenoble : thesebum@ujf-grenoble.fr

\section{LIENS}

Code de la Propriété Intellectuelle. articles $L$ 122. 4

Code de la Propriété Intellectuelle. articles L 335.2- L 335.10 http://www.cfcopies.com/V2/leg/leg droi.php http://www.culture.gouv.fr/culture/infos-pratiques/droits/protection.htm 
UNIVERSITE JOSEPH FOURIER

FACULTE DE MEDECINE DE GRENOBLE

Année : 2012

$\mathrm{N}^{\circ}$

RAIVE (RhumAtologie InterVentionnelle et Echographie) : résultats d'une enquête nationale.

THESE

PRESENTEE POUR L'OBTENTION DU DOCTORAT EN MEDECINE

DIPLÔME D’ETAT

Aurélie Sicaud

Née le 12/02/1983

A Bourgoin-Jallieu (38)

THESE SOUTENUE PUBLIQUEMENT A LA FACULTE DE MEDECINE DE GRENOBLE*

Le 22 octobre 2012

DEVANT LE JURY COMPOSE DE

Président : M. le Professeur R. Juvin

Membres: M. le Professeur P. Gaudin (Directeur de thèse)

M. le Professeur D. Saragaglia

M. le Docteur D. Briançon

* La Faculté de Médecine de Grenoble n'entend donner aucune approbation ni improbation aux opinions émises dans les thèses; ces opinions sont considérées comme propres à leurs auteurs. 


\section{Tables des matières :}

Liste des Professeurs des Universités

..p.4

Liste des Maîtres de Conférence des Universités. .p. 7

Remerciements ..p.9

Justification de l'étude. p.11

Résumé .p.12

Introduction .p.13

Méthodes p.14

Résultats p.15

Discussion p.19

Conclusion p.22

Bibliographie p. 23

Annexe : questionnaire p.26

Permis d'impression p.28

Serment d'Hippocrate p.29 
Professeur des Universités - Praticien Hospitalier

2011-2012

\begin{tabular}{|c|c|c|}
\hline Nom & Prénom & Intitulé de la discipline universitaire \\
\hline ALBALADEJO & Pierre & Anesthésiologie-réanimation \\
\hline ARVIEUX-BARTHELEMY & Catherine & Chirurgie générale \\
\hline BACONNIER & Pierre & $\begin{array}{c}\text { Biostatiques, informatique médicale et technologies de } \\
\text { communication }\end{array}$ \\
\hline BAGUET & Jean-Philippe & Cardiologie \\
\hline BALOSSO & Jacques & Radiothérapie \\
\hline BARRET & Luc & Médecine légale et droit de la santé \\
\hline BAUDAIN & Philippe & Radiologie et imagerie médicale \\
\hline BEANI & Jean-Claude & Dermato-vénéréologie \\
\hline BENHAMOU & Pierre Yves & Endocrinologie, diabète et maladies métaboliques \\
\hline BERGER & François & Biologie cellulaire \\
\hline BLIN & Dominique & Chirurgie thoracique et cardio-vasculaire \\
\hline BOLLA & Michel & Cancérologie; radiothérapie \\
\hline BONAZ & Bruno & Gastroentérologie; hépatologie; addictologie \\
\hline BOSSON & Jean-Luc & $\begin{array}{c}\text { Biostatiques, informatique médicale et technologies de } \\
\text { communication }\end{array}$ \\
\hline BOUGEROL & Thierry & Psychiatrie d'adultes \\
\hline BRAMBILLA & Elisabeth & Anatomie et cytologie pathologiques \\
\hline BRAMBILLA & Christian & Pneumologie \\
\hline BRICAULT & Ivan & Radiologie et imagerie médicale \\
\hline BRICHON & Pierre-Yves & Chirurgie thoracique et cardio-vasculaire \\
\hline BRIX & Muriel & Chirurgie maxillo-faciale et stomatologie \\
\hline CAHN & Jean-Yves & Hématologie \\
\hline CARPENTIER & Françoise & Thérapeutique; médecine d'urgence \\
\hline CARPENTIER & Patrick & Chirurgie vasculaire; médecine vasculaire \\
\hline CESBRON & Jean-Yves & Immunologie \\
\hline CHABARDES & Stephan & Neurochirurgie \\
\hline CHABRE & Olivier & Endocrinologie, diabète et maladies métaboliques \\
\hline CHAFFANJON & Philippe & Anatomie \\
\hline CHAVANON & Olivier & Chirurgie thoracique et cardio-vasculaire \\
\hline CHIQUET & Christophe & Ophtalmologie \\
\hline CHIROSSEL & Jean-Paul & Anatomie \\
\hline CINQUIN & Philippe & $\begin{array}{c}\text { Biostatiques, informatique médicale et technologies de } \\
\text { communication }\end{array}$ \\
\hline COHEN & Olivier & $\begin{array}{c}\text { Biostatiques, informatique médicale et technologies de } \\
\text { communication }\end{array}$ \\
\hline COUTURIER & Pascal & Gériatrie et biologie du vieillissement \\
\hline CRACOWSKI & Jean-Luc & Pharmacologie fondamentale; pharmacologie clinique \\
\hline
\end{tabular}




\begin{tabular}{|c|c|c|}
\hline DE GAUDEMARIS & Régis & Médecine et santé au travail \\
\hline DEBILLON & Thierry & Pédiatrie \\
\hline DEMATTEIS & Maurice & Addictologie \\
\hline DEMONGEOT & Jacques & $\begin{array}{c}\text { Biostatiques, informatique médicale et technologies de } \\
\text { communication }\end{array}$ \\
\hline DESCOTES & Jean-Luc & Urologie \\
\hline ESTEVE & François & Biophysique et médecine nucléaire \\
\hline FAGRET & Daniel & Biophysique et médecine nucléaire \\
\hline FAUCHERON & Jean-Luc & Chirurgie générale \\
\hline FERRETTI & Gilbert & Radiologie et imagerie médicale \\
\hline FEUERSTEIN & Claude & Physiologie \\
\hline FONTAINE & Eric & Nutrition \\
\hline FRANCOIS & Patrice & Epidémiologie, économie de la santé et prévention \\
\hline GARBAN & Frédéric & Hématologie; transfusion \\
\hline GAUDIN & Philippe & Rhumatologie \\
\hline GAVAZZI & Gaetan & Gériatrie et biologie du vieillissement \\
\hline GAY & Emmanuel & Neurochirurgie \\
\hline GRIFFET & Jacques & Chirurgie infantile \\
\hline HALIMI & Serge & Nutrition \\
\hline HOMMEL & Marc & Neurologie \\
\hline JOUK & Pierre-Simon & Génétique \\
\hline JUVIN & Robert & Rhumatologie \\
\hline KAHANE & Philippe & Physiologie \\
\hline KRACK & Paul & Neurologie \\
\hline KRAINIK & Alexandre & Radiologie et imagerie médicale \\
\hline LANTUEJOUL & Sylvie & Anatomie et cytologie pathologiques \\
\hline LEBAS & Jean-François & Biophysique et médecine nucléaire \\
\hline LEBEAU & Jacques & Chirurgie maxillo-faciale et stomatologie \\
\hline LECCIA & Marie-Thérèse & Dermato-vénéréologie \\
\hline LEROUX & Dominique & Génétique \\
\hline LEROY & Vincent & Gastroentérologie; hépatologie; addictologie \\
\hline LETOUBLON & Christian & Chirurgie générale \\
\hline LEVY & Patrick & Physiologie \\
\hline LUNARDI & Joël & Biochimie et biologie moléculaire \\
\hline MACHECOURT & Jacques & Cardiologie \\
\hline MAGNE & Jean-Luc & Chirurgie vasculaire \\
\hline MAITRE & Anne & Médecine et santé au travail \\
\hline MAURIN & Max & Bactériologie-virologie \\
\hline MERLOZ & Philippe & Chirurgie orthopédique et traumatologique \\
\hline
\end{tabular}




\begin{tabular}{|c|c|c|}
\hline MORAND & Patrice & Bactériologie-virologie \\
\hline MORO-SIBILOT & Denis & Pneumologie \\
\hline MOUSSEAU & Mireille & Cancérologie \\
\hline MOUTET & François & Chirurgie plastique, reconstructrice et esthétique; brûlogie \\
\hline PALOMBI & Olivier & Anatomie \\
\hline PASSAGIA & Jean-Guy & Anatomie \\
\hline PAYEN DE LA GARANDERIE & Jean-François & Anesthésiologie-réanimation \\
\hline PELLOUX & Hervé & Parasitologie et mycologie \\
\hline PEPIN & Jean-Louis & Physiologie \\
\hline PERENNOU & Dominique & Médecine physique et de réadaptation \\
\hline PERNOD & Gilles & Médecine vasculaire \\
\hline PIOLAT & Christian & Chirurgie infantile \\
\hline PISON & Christophe & Pneumologie \\
\hline PLANTAZ & Dominique & Pédiatrie \\
\hline POLACK & Benoît & Hématologie \\
\hline PONS & Jean-Claude & Gynécologie-obstétrique \\
\hline RAMBEAUD & Jean-Jacques & Urologie \\
\hline REYT & Emile & Oto-rhino-laryngologie \\
\hline RIGHINI & Christian & Oto-rhino-laryngologie \\
\hline ROMANET & Jean-Paul & Ophtalmologie \\
\hline SARAGAGLIA & Dominique & Chirurgie orthopédique et traumatologique \\
\hline SCHMERBER & Sébastien & Oto-rhino-laryngologie \\
\hline SELE & Bernard & Biologie et médecine du développement et de la reproduction \\
\hline SERGENT & Fabrice & Gynécologie-obstétrique \\
\hline SESSA & Carmine & Chirurgie vasculaire \\
\hline STAHL & Jean-Paul & Maladies infectueuses; maladies tropicales \\
\hline STANKE & Françoise & Pharmacologie fondamentale \\
\hline TIMSIT & Jean-François & Réanimation \\
\hline TONETTI & Jérôme & Chirurgie orthopédique et traumatologique \\
\hline TOUSSAINT & Bertrand & Biochimie et biologie moléculaire \\
\hline VANZETTO & Gérald & Cardiologie \\
\hline VUILLEZ & Jean-Philippe & Biophysique et médecine nucléaire \\
\hline WEIL & Georges & Epidémiologie, économie de la santé et prévention \\
\hline ZAOUI & Philippe & Néphrologie \\
\hline ZARSKI & Jean-Pierre & Gastroentérologie; hépatologie; addictologie \\
\hline
\end{tabular}


Mâ̂tre de Conférence des Universités - Praticien Hospitalier

2011-2012

\begin{tabular}{|c|c|c|}
\hline Nom & Prénom & Intitulé de la discipline universitaire \\
\hline BONNETERRE & Vincent & Médecine et santé au travail \\
\hline BOTTARI & Serge & Biologie cellulaire \\
\hline BOUTONNAT & Jean & Cytologie et histologie \\
\hline BRENIER-PINCHART & Marie-Pierre & Parasitologie et mycologie \\
\hline BRIOT & Raphaël & Thérapeutique; médecine d'urgence \\
\hline CALLANAN-WILSON & Mary & Hématologie; transfusion \\
\hline CROIZE & Jacques & Bactériologie-virologie \\
\hline DERANSART & Colin & Physiologie \\
\hline DETANTE & Olivier & Neurologie \\
\hline DUMESTRE-PERARD & Chantal & Immunologie \\
\hline EYSSERIC & Hélène & Médecine légale et droit de la santé \\
\hline FAURE & Julien & Biochimie et biologie moléculaire \\
\hline GILLOIS & Pierre & $\begin{array}{c}\text { Biostatiques, informatique médicale et technologies de } \\
\text { communication }\end{array}$ \\
\hline GRAND & Sylvie & Radiologie et imagerie médicale \\
\hline HENNEBICQ & Sylviane & Biologie et médecine du développement et de la reproduction \\
\hline HOFFMANN & Pascale & Gynécologie-obstétrique \\
\hline LABARERE & José & Epidémiologie, économie de la santé et prévention \\
\hline LAPORTE & François & Biochimie et biologie moléculaire \\
\hline LARDY & Bernard & Biochimie et biologie moléculaire \\
\hline LARRAT & Sylvie & Bactériologie-virologie \\
\hline LAUNOIS-ROLLINAT & Sandrine & Physiologie \\
\hline MALLARET & Marie-Reine & Epidémiologie, économie de la santé et prévention \\
\hline MAUBON & Danièle & Parasitologie et mycologie \\
\hline MC LEER (FLORIN) & Anne & Cytologie et histologie \\
\hline MOREAU-GAUDRY & Alexandre & $\begin{array}{c}\text { Biostatiques, informatique médicale et technologies de } \\
\text { communication }\end{array}$ \\
\hline MOUCHET & Patrick & Physiologie \\
\hline
\end{tabular}




\begin{tabular}{|c|c|c|}
\hline PACLET & Marie-Hélène & Biochimie et biologie moléculaire \\
\hline PASQUIER & Dominique & Anatomie et cytologie pathologiques \\
\hline PAYSANT & François & Médecine légale et droit de la santé \\
\hline PELLETIER & Laurent & Biologie cellulaire \\
\hline RAY & Pierrétique \\
\hline RIALLE & Vincent & Biostatiques, informatique médicale et technologies de \\
\hline SATRE & Véronique & Génétique \\
\hline STASIA & Marie-Josée & Biochimie et biologie moléculaire \\
\hline TAMISIER & Renaud & Physiologie \\
\hline
\end{tabular}




\section{Remerciements :}

J'adresse mes remerciements à tous ceux qui m'ont aidé tout au long de mes études de médecine et plus particulièrement lors de la réalisation de ma thèse :

- Monsieur le Professeur Juvin, je vous remercie d'avoir accepté de présider ce jury et de me permettre de poursuivre mon chemin au sein de votre équipe lors des deux prochaines années... Merci pour vos visites toujours aussi captivantes et passionnantes...

- Monsieur le Professeur Gaudin, Directeur de thèse, je vous remercie de m'avoir confiée ce travail, de m'avoir conseillée et d'avoir été présent durant mon internat. Merci pour ces moments de bonne humeur et très formateurs en salle interventionnelle...

- Monsieur le Professeur Saragaglia, je vous remercie d'avoir accepté de participer à ce jury et de m'avoir fait confiance pour l'étude de vos dossiers afin de réaliser un fabuleux travail sur la prise en charge des infections de prothèse...

- Monsieur le Docteur Briançon, je vous remercie de me faire l'honneur d'être dans ce jury et de m'avoir accueillie dans votre service. Votre bonne humeur, votre humour et votre douceur au quotidien sont un régal. Etes-vous sûr de vouloir prendre votre retraite si tôt ?

- Merci à mes assistantes de rhumatologie pour leur qualité pédagogique et leur présence : Dana, Mélanie, Anne et Alexandra. Merci à Laurent pour sa bonne humeur et que serions-nous sans ses blagues?

- Merci aux dream-teams d'infectiologie du CH de Chambéry (Cécile, Dorothée, Claire, Emmanuel, et le chef avec son sourire légendaire : Dc Rogeaux...) et du CHU de Grenoble (Virginie, Sandrine, Dc JP Brion et son charisme inimitable, Pr Stahl, et Patricia, mon idole, qui m'a fait découvrir et aimer l'infectiologie, merci pour ton aide et ta disponibilité...) 
- Merci à Olivier pour sa présence et ses conseils. L'aventure commence...

- Merci aux infirmières, aides-soignantes, kiné, secrétaires, assistantes sociales ... de rhumatologie du CHU de Grenoble et de la Reine Hortense, d'infectiologie du CHU et du $\mathrm{CH}$ Chambéry, de MPR vasculaire du CHU et de neurologie du CHR Annecy...

- Merci à mes parents, à ma sœur, à mon beau frère et à toute ma famille de m'avoir accompagnée et soutenue durant toutes mes longues études. Merci de m'avoir inculquée ses valeurs qui aujourd'hui sont les miennes... Une pensées pour ceux qui me regarde de là-haut...

- Je fais d'énormes poutoupoutous à ma louloute Elïn, ma nièce, en attendant son petit frère...

- Merci à tous mes amis et co -internes. Cette fois-ci c'est promis, je vais être plus disponible pour faire la fiesta, des rando et quelques voyages : Cécile, Maja (et sa légendaire Vodka Tabasco made in Pologne), Thomas et Elyse, Thérèse, Stéphanie, Nancy et Xavier, Joseph, Charlotte (ma géniale binôme du B7), François (et sa zen attitude), Caroline (ma super co-interne de RH), Florence (toujours présente pour déboucher une bonne bouteille au bord du lac), Aurélie et Vincent, Caroline et Jérôme, Amandine et Didier, les guerrières (Cochon, la Gone Caro, Rach et le coach Georgeounnet)...

- Mme Nathalie Tobal du laboratoire Chugai et Mme Chantal Rousseaux de la Société Nukléus pour leur soutien. 


\section{Justification de l'étude}

Depuis plus de 10 ans, l'échographie musculo-squelettique a pris une place considérable dans la pratique de la rhumatologie aussi bien sur le plan diagnostique que thérapeutique avec l'échographie interventionnelle. Plusieurs pays comme l'Angleterre, l'Irlande ou encore les Etats-Unis d'Amérique ont déjà publié un état des lieux concernant leurs pratiques et leurs formations en échographie rhumatologique. Actuellement, en France, aucune étude n'a été réalisée sur ce thème. Nous avons donc souhaité faire une synthèse concernant la pratique de l'échographie musculo-squelettique interventionnelle dans notre pays afin d'identifier les points positifs et négatifs ainsi que les besoins des médecins afin d'améliorer notre pratique qui s'inscrit comme une spécialité au sein de la rhumatologie.

Cette thèse est rédigée sous la forme d'un article. Cet article est en cours de soumission pour la Revue du Rhumatisme et est présenté tel qu'il a été adressé à l'éditeur. 


\section{Résumé :}

Objectif. Décrire la place de l'échographie interventionnelle dans l'activité des rhumatologues français.

Méthodes. Un questionnaire concernant les formations, la pratique, les conditions de réalisation, les aspects économiques a été envoyé à 3412 rhumatologues, médecins du sport et rééducateurs fonctionnels exerçant en France.

Résultats. 388 questionnaires (11,3\%) ont été analysés entre le 20/09 et le 16/11 2011. $326(84.2 \%)$ des participants sont rhumatologues libéraux (36.1\%), hospitaliers (36.4\%) ou mixte (27.5\%). 201 (51.7\%) déclarent être formé à l'échographie. La médiane d'année de certification est 2008 (1996 -2011). 227 ont accès à un échographe qui est renouvelé en moyenne tous les 4.8 ans $( \pm 1.3)$.

Les indications de l'échographie sont toutes les pathologies musculo-squelettiques (50.9\%) et les rhumatismes inflammatoires (30.4\%) principalement. 154 (40\%) réalisent au moins une échographie interventionnelle par semaine avec une moyenne hebdomadaire de 7.8 \pm 8.1 gestes. L'antisepsie est réalisée en 5 temps (recommandations HAS) par $49.7 \%$ d'entre eux. Gel et housse stériles sont utilisés respectivement par $67.9 \%$ et $61 \% .27 .7 \%$ pratiquent uniquement l'écho repérage, $10.1 \%$ autant d'écho guidage que d'écho repérage.

$74.5 \%$ cotent le geste mais $71.7 \%$ pensent que l'échographie musculo-squelettique interventionnelle n'est pas rentable.

$67.1 \%$ souhaitent bénéficier d'une formation complémentaire principalement en échographie interventionnelle.

Conclusion. L'échographie musculo-squelettique interventionnelle et diagnostique sont de plus en plus pratiquée en France. Les médecins ont un fort souhait de formation.

Mots clés: échographie musculo-squelettique; infiltration; formation; antisepsie ; aspect économique. 


\section{Introduction}

Depuis le début des années 2000, l'échographie musculo-squelettique (EMS) connait un intérêt croissant d'abord en Europe [1,2] puis aux Etats-Unis [3,4] aussi bien en pratique clinique rhumatologique qu'en recherche. Plusieurs études ont démontré la supériorité de l'EMS par rapport à l'examen clinique seul [5] pour la mise en évidence des petits épanchements articulaires [6], des synovites [7,8], des érosions et des pathologies microcristallines [9]. L'EMS permet un diagnostic plus précoce et une prise en charge thérapeutique plus rapide que l'examen clinique [10]. Comme dans de nombreuses spécialités qui utilisent les ultrasons en soutien de la clinique [11], la rhumatologie a su employer cette technique pour améliorer la prise en charge diagnostique et thérapeutique des patients.

Plus récemment, l'EMS a été utilisée en rhumatologie interventionnelle. La plupart des gestes extra-rachidiens (ponction, infiltrations articulaires, péri-articulaires et péritendineuses, biopsies...), réalisés jusqu'à présent par repérages anatomiques ou radioscopiques, sont réalisables sous échographie. Plus récemment encore, ont été décrites des infiltrations rachidiennes et sacro-iliaques [12].

Deux techniques sont pratiquées: l'écho-guidage et l'écho-repérage. L'utilisation des ultrasons (US) pour la réalisation des infiltrations améliore la précision des gestes [13] et leur efficacité [14] aussi bien au niveau des grosses articulations comme l'épaule $[15,16]$ ou le genou [17], les moyennes articulations [18,19] et les petites articulations [20]. L'objectif de cette étude est de décrire la place de l'échographie interventionnelle musculo-squelettique dans l'activité des rhumatologues français. 


\section{Méthodes}

\subsection{Population}

L'enquête réalisée est une étude observationnelle, transversale et nationale. Un questionnaire a été envoyé en septembre 2011 à 2486 rhumatologues, 905 rééducateurs et 21 médecins du sport, soit 3412 médecins au total (base de données société Nukléus). Ils exercent soit en milieu hospitalier, soit en libéral soit ont une activité mixte. Une enveloppe $T$ était jointe au questionnaire afin de faciliter les réponses.

\subsection{Questionnaire}

Le questionnaire comporte 25 questions traitant de la formation, de la pratique, des procédures d'antisepsie et des aspects économiques en termes d'EMS interventionnelle. La première partie du questionnaire étudie la description de la population : spécialité (rhumatologie, médecine du sport, médecine physique et de réadaptation), type d'activité (libérale, hospitalière, mixte) et ancienneté de la pratique.

La seconde partie traite de la formation reçue pour pratiquer l'échographie: type de diplôme, année de certification à l'échographie.

La troisième partie analyse la pratique de l'EMS interventionnelle et est divisée en plusieurs paragraphes: échographe (accessibilité, référence de la machine, achat ou location, période de renouvellement), organisation (local dédié, plage horaire, aide), méthodes (échoguidage, écho repérage, radioscopie), indications.

La quatrième partie concerne l'antisepsie réalisée au cours des gestes sous échographie : antisepsie type HAS en 5 temps ou non, produit utilisé, housse stérile, gel stérile, complications.

La cinquième partie intéresse les aspects économiques (nombre de gestes réalisés sous échographie par semaine, temps moyen par geste, cotation, rentabilité de l'échographie interventionnelle).

La dernière partie traite des souhaits de formation à l'échographie interventionnelle : délai, type de formation à l'échographie.

\subsection{Analyse statistique}

Les différentes variables descriptives sont exprimées soit sous la forme de pourcentage soit sous la forme de moyenne \pm écart type soit sous la forme de médiane. 


\section{Résultats}

\subsection{Population}

Le questionnaire a été envoyé en septembre 2011 à 3412 médecins spécialistes exerçant en France. 388 questionnaires (11.3\%) ont été retournés entre le 20 septembre et le 16 novembre 2011. Parmi les 388 médecins participants, 326 sont rhumatologues (84.2\%), 37 sont rééducateurs (9.6\%), 11 sont rhumatologues et rééducateurs (2.8\%), 7 sont médecins du sport et rééducateurs (1.8\%), 3 sont médecins du sport $(0.8 \%), 2$ exercent les trois disciplines (0.5\%), 1 est rhumatologue et rééducateur (0.3\%) et 1 n'a pas renseigné sa spécialité $(0.3 \%)$. La médiane de l'année de soutenance de thèse est 1989 (1958 - 2010). La répartition est homogène entre activité libérale (36.1\%), hospitalière (36.4\%) et mixte (27.5\%).

\subsection{Formation à l'échographie interventionnelle}

82 médecins déclarent ne plus faire d'échographie. 201 (66.56\%) ont reçu au moins une formation pour la pratique de l'échographie. 101 médecins ont participé au DU ECRIN, 45 à Echorhum, 11 au DIU d'Echographie et de Techniques ultrasonores, 4 au DU REGARDE, 9 à plusieurs formations et 31 à au moins une autre formation.

La médiane de l'année de la certification est 2008 avec des extrêmes variant de 1996 à 2011. 3 médecins étaient en cours de formation.

67.1\% des médecins ayant répondu à l'enquête souhaiteraient avoir une formation complémentaire. Parmi eux, 67.9\% aimeraient bénéficier d'une formation à l'échographie avec une application interventionnelle, $19.3 \%$ à l'échographie et à la radiologie interventionnelle, $8.6 \%$ à l'échographie seule et $4.3 \%$ à la radiologie interventionnelle seule. $50.9 \%$ des médecins envisagent de faire une formation dans les 2 prochaines années.

\subsection{Pratique de l'échographie interventionnelle}

\subsubsection{L'échographe}

227 médecins (58.5\%) déclarent avoir accès à un échographe. Parmi eux, 89 (39.2\%) utilisent l'échographe dans leur service hospitalier, 65 (28.6\%) ont leur échographe personnel, 16 (7.1\%) disposent d'un échographe dans un cabinet de groupe, 13 (5.7\%) ont accès à l'échographe du service hospitalier de radiologie, 4 (1.8\%) ont une vacation chez un radiologue libéral et 40 (17.6\%) ont recours à un échographe selon au moins deux 
accès parmi ceux cités précédemment. Les marques de l'échographe utilisé par les médecins participants sont Esaote ${ }^{\circledR}$ (55.6\%), General Electric ${ }^{\circledR}$ (10.5\%), Toshiba ${ }^{\circledR}$ (9.9\%), Sonosite $^{\circledR}$ (6.4\%), Hitachi ${ }^{\circledR}$ (4.7\%), Philips ${ }^{\circledR}$ (4.1\%), BK ${ }^{\circledR}$ (2.9\%), Siemens ${ }^{\circledR} \quad$ (2.3\%) et autres marques (3.5\%). 145 (74.4\%) médecins parmi ceux ayant accès à un appareil d'échographie l'ont acheté, 21 (10.8\%) louent l'échographe, 25 (12.8\%) ont un échographe mis à disposition, $3(1.5 \%)$ bénéficient d'échographes achetés et mis à disposition et $1(0.5 \%)$ médecin dispose d'un échographe acheté et un loué. L'échographe est renouvelé en moyenne tous les 4.8 ans \pm 1.3 .

\subsubsection{Organisation}

54.5\% des médecins ayant répondu au questionnaire ont un local ou une salle dédiée à la pratique de l'EMS interventionnelle. $67.6 \%$ sont seuls pour réaliser les gestes sous échographie, $28.5 \%$ se font aider par une tierce personne et $3.9 \%$ travaillent seuls ou se font aider. $44.1 \%$ de ceux pratiquant l'échographie interventionnelle ont des plages horaires dédiées à cette pratique. 55.1\% réalisent des gestes avec l'aide de l'échographie au cours de la consultation.

\subsubsection{Méthodes}

22.9\% des médecins interrogés utilisent exclusivement l'échographie pour la réalisation des gestes (ponction et/ou infiltration), 23.2\% utilisent exclusivement la radiologie. 33.6\% réalisent les gestes en employant l'une ou l'autre imagerie. Le guidage est uniquement clinique chez $14.4 \%$. Certains appliquent une combinaison guidage clinique et échographique (3.3\%) ou guidage clinique et radioscopique $(0.7 \%)$ ou guidage clinique et l'une ou l'autre imagerie (1.8\%). La répartition des gestes réalisés soit par échorepérage soit par échoguidage est représentée sur la figure 1.

\section{Figure 1}

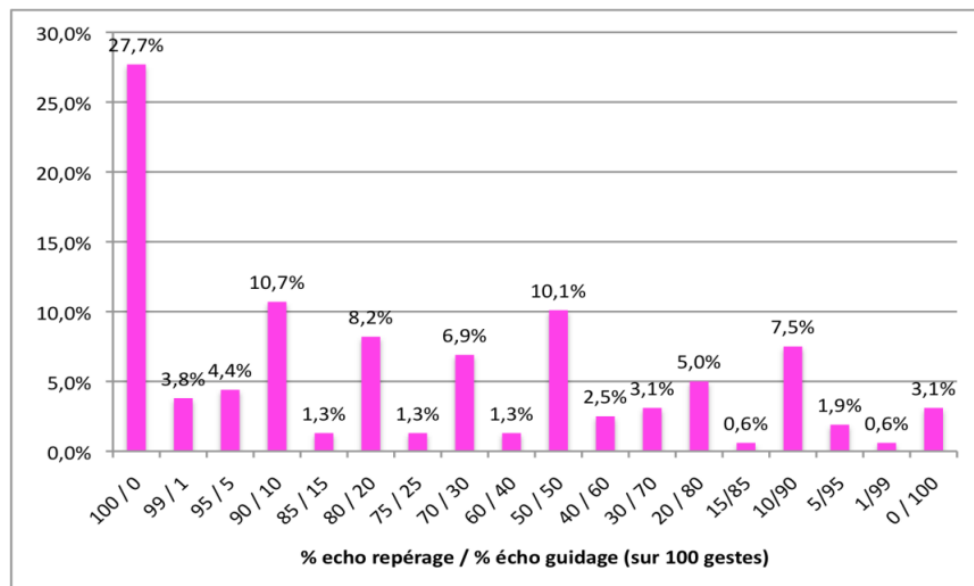


Cette répartition est expliquée par la rapidité du geste sous écho-repérage (33.3\%), l'absence d'aide disponible (22.7\%), d'autres causes (21.3\%) ou par la combinaison de 2 ou 3 de ces raisons (22.6\%).

\subsubsection{Indications}

Les indications de la réalisation d'une échographie interventionnelle sont représentées sur la figure 2.

Figure 2

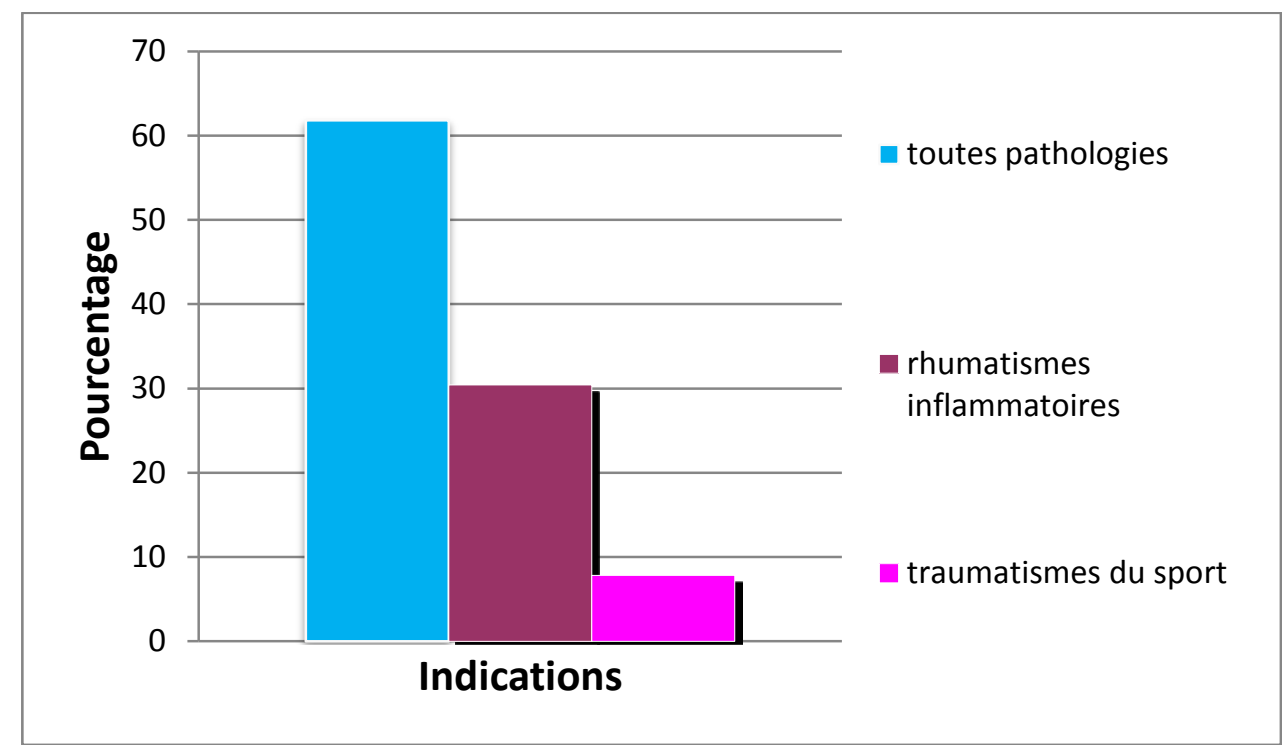

\subsubsection{Aspect économique}

154 déclarent faire au moins une échographie interventionnelle par semaine. Ils pratiquent en moyenne $8.6 \pm 9.5$ gestes par semaine. Le temps moyen consacré par geste sous échographie est de $18.6 \pm 8.6$ minutes. Les temps extrêmes vont de 2 à 60 minutes. 74.5\% cotent les échographies interventionnelles lors de la facturation. $71.1 \%$ pensent que cette activité de la rhumatologie interventionnelle n'est pas rentable financièrement. 


\subsection{Antisepsie}

155 médecins ont répondu aux questions sur l'antisepsie. 49.7\% d'entre eux réalisent l'antisepsie selon les 5 temps de l'HAS. 81.3\% utilisent de la povidone iodée (Bétadine ${ }^{\circledR}$ ). 18.7\% (soit 29 médecins) emploient d'autres produits pour la désinfection (tableau 2).

\begin{tabular}{llll} 
& Produit utilisé & $\begin{array}{l}\text { Effectif } \\
\mathbf{n = 2 9}\end{array}$ & \% \\
\cline { 2 - 4 } & $\begin{array}{l}\text { Données } \\
\text { manquantes }\end{array}$ & 2 & \\
Tableau 2 & $\begin{array}{l}\text { Chlorhexidine } \\
\text { Alcool }\end{array}$ & 16 & $59.4 . \%$ \\
& Hexomedine ${ }^{\circledR}$ & 9 & $33,3 \%$ \\
& 2 & $7,4 \%$ \\
\hline
\end{tabular}

Le gel stérile et les housses stériles pour la sonde sont utilisés respectivement par $67.9 \%$ et $61 \%$ des médecins. Une seule infection iatrogène a été déclarée. 


\section{Discussion}

\subsection{Formation : de l'essentielle théorie à la mise en application...}

Dans notre étude, $66.58 \%$ des rhumatologues qui pratiquent l'EMS ont effectué au moins une formation. La majorité a validé un diplôme universitaire d'une durée d'un an. Ce taux de formation est supérieur aux données publiées par l'EULAR ou le taux de formation des rhumatologues français était compris entre 10 et $50 \%$ à la fin de l'année 2009 et début de l'année 2010 [22]. Seule l'Espagne avait un taux de formation à l'EMS supérieur à 50\%. En France, la formation à l'échographie est structurée sous la forme de diplômes universitaires associant des cours théoriques et pratiques. L'EULAR a émis des recommandations en 2007 sur l'organisation de la formation à l'EMS en Europe [23]. Un modèle de formation en 3 niveaux est proposé : basique, intermédiaire et avancé. Cette éducation en trois niveaux s'effectue sur des cycles d'une durée minimum de 2 ans. Chaque niveau permet d'acquérir des compétences supplémentaires. L'accès au premier niveau est ouvert à tous les rhumatologues. Le passage au niveau supérieur nécessite d'avoir pratiqué un nombre d'échographie minimum : 100 échographies pour l'accès au niveau intermédiaire et 300 pour le niveau avancé. Les anglais ont établi un cadre éducatif (" educational framework") afin de faciliter la formation à l'échographie des rhumatologues [24-27]. Les irlandais ont également créé un programme de formation à la pratique de l'échographie en rhumatologie. Ce programme est organisé en plusieurs modules de niveau croissant [28]. Toutes ces restructurations de l'organisation du système de formation à l'EMS partent du constat du manque de formation, d'uniformisation et de reconnaissance de la formation à l'échographie en rhumatologie $[29,30]$. D'autres pays comme le Canada [4] ou la Roumanie [31] sont plus en retard concernant leur système de formation à la pratique de l'échographie. La plupart des programmes mis en place en Europe [22,27] et aux Etats Unis d'Amérique [3] concerne principalement l'échographie diagnostique. L'échographie interventionnelle semble peu enseignée dans ces différents programmes. Or dans notre étude, deux tiers des rhumatologues interrogés souhaitent bénéficier $d$ 'une formation supplémentaire en échographie musculo-squelettique, et $87 \%$ d'entre eux désirent plus particulièrement une formation à l'échographie interventionnelle. La moitié de ces derniers veulent faire cette formation dans les deux prochaines années. En France, la formation à l'échographie musculo-squelettique est universitaire [32]. Le diplôme interuniversitaire (DIU) d'Echographie et Techniques ultrasonores de l'université René-Descartes à Paris-V et le diplôme universitaire (DU) ECRIN de l'université Pierre-et-Marie-Curie à Paris-VI, créé en 2006, sont plus axés sur le diagnostic échographique que sur la réalisation des gestes sous échographie. En 2010, le DU REGARDE de l'université Joseph-Fourier à Grenoble a été créé et est consacré à la rhumatologie interventionnelle avec option échographie seule ou échographie et radioscopie. En 2012, le DIU de rhumatologie interventionnelle 
échographique de l'université de Nantes a été modifié afin d'assurer une formation exclusive à l'échographie interventionnelle. Ces évolutions des programmes d'apprentissage sont nécessaires pour pouvoir répondre aux demandes des rhumatologues. La formation à l'EMS appliquée à l'interventionnelle devrait faire partie des compétences nécessaires pour la validation du diplôme d'étude spécialisé de rhumatologie.

\subsection{Pratique de l'échographie : une nécessité de réorganisation...}

Parmi les rhumatologues pratiquant l'échographie, la moitié d'entre eux (50.9\%) utilise les ultrasons pour les infiltrations articulaires et péri-articulaires. Ce taux est supérieur aux données de l'étude de l'EULAR de Mandl et al [22] ou 30\% des répondeurs utilisaient l'EMS pour les infiltrations ou les ponctions et $12 \%$ pour les articulations profondes (hanche, épaule) [33]. La technique de l'écho-repérage est plus souvent utilisée que l'écho-guidage car c'est une technique plus rapide à réaliser, qui ne nécessite ni recours à une tierce personne ni utilisation d'une housse et de gel stérile étant donné que la désinfection est effectuée après la réalisation de l'échographie. II faut également souligner l'implication des rhumatologues français pour cette pratique puisque que la moitié d'entre eux ont un local et des plages horaires dédiés à la réalisation des gestes sous échographie.

\subsection{Antisepsie : indispensable mais consommant du temps !}

Près de la moitié des rhumatologues interrogés réalisent l'antisepsie selon les 5 temps recommandés par la Haute Autorité de Santé (HAS). Cet acte d'antisepsie comme il est décrit dans les recommandations actuelles commence par un nettoyage local, suivi d'un rinçage à l'eau, puis d'un séchage avant le badigeonnage de l'antiseptique et enfin un séchage. La povidone iodée (Bétadine ${ }^{\circledR}$ ) est l'antiseptique le plus utilisé (81.3\%) pour I'antisepsie avant la réalisation d'un geste sous contrôle échographique. La chlorhexidine (Biseptine ${ }^{\circledR}, \mathrm{Hibiscrub}^{\circledR}, \mathrm{Cyteal}^{\circledR}, \mathrm{Hibitane}^{\circledR} \ldots$ ) et l'alcool sont également utilisés. L'hexamidine (Hexomédine ${ }^{\circledR}$ ) est employée par 2 rhumatologues mais n'est pas recommandée pour l'antisepsie avant ponction cutanée en raison de son délai d'action trop long. Une seule infection survenue après une infiltration a été rapportée, bien que la moitié des rhumatologues utilisent un autre protocole d'antisepsie que celui recommandé par l'HAS. L'acte d'antisepsie comme il est recommandé actuellement est long. Ceci peut rendre son application stricte difficile en pratique courante et notamment en libéral. Des études sur les autres procédures d'antisepsie comparée au protocole de référence en 5 temps devront être réalisées. Si d'autres procédures de désinfection plus courtes et aussi efficaces pouvaient être validées, ceci faciliterait la réalisation des gestes sous contrôle échographique. L'utilisation de housse stérile pour la sonde d'échographie 
et de gel stérile est plus fréquente en France dans notre étude que dans les autres pays européens [22] bien qu'il n'existe actuellement aucune recommandation officielle quant à leur utilisation.

\subsection{Aspect économique : une technique intéressante à but non lucratif !}

Un des freins à la pratique de l'échographie musculo-squelettique interventionnelle est l'accessibilité à un échographe. Même si le parc échographique est en constante augmentation depuis la dernière décade, il reste encore $42 \%$ des rhumatologues interrogés qui ne disposent d'aucun accès à un échographe. Le coût d'un appareil d'échographie équipé de sonde haute fréquence $(5-18 \mathrm{MHz})$ est élevé et représente un investissement conséquent aussi bien en structure hospitalière qu'en libérale. Le prix d'un tel équipement est compris entre $20000 €$ et $130000 €$ [34], auquel il faut rajouter le coût de la maintenance. En France et en 2012, plusieurs codages existent pour une échographie articulaire : PCQM001 : échographie de muscle et/ou de tendon ; PBQM001 : échographie unilatérale ou bilatérale de plusieurs articulations; PBQM002 : échographie unilatérale ou bilatérale d'une articulation; PBQM003: échographie unilatérale ou bilatérale d'une articulation et de son appareil capsulo-ligamentaire; PBQM004: échographie unilatérale ou bilatérale de plusieurs articulations et de leur appareil capsulo-ligamentaire. Mais tous ces codages correspondent à la somme unique de $37.80 €$ quelque soit le nombre d'articulations étudiées. Un geste écho-guidé est moins bien rémunéré qu'un geste écho-repéré (ex : articulation du membre supérieur : guidage $=$ YYYY028 + 1/2 ZZL002 (soit 42.60€) versus repérage= PBQM002 + 1/2 MZLB001 (soit $50.86 €)$. Un nombre important d'infiltration est donc nécessaire afin de pouvoir amortir cet investissement. Mais sachant que le temps moyen d'un geste sous échographie est de 18 minutes et peut aller jusqu'à une heure, il est difficile de multiplier le nombre de gestes dans la pratique quotidienne. Ceci explique le sentiment de $71.1 \%$ des rhumatologues français interrogés qui pensent que cette activité n'est pas rentable financièrement.

Les limites de cette étude sont le taux de réponse de $11 \%$ et le nombre de données manquantes alors que les réponses étaient principalement sous la forme de cases à cocher et qu'il y avait très peu de questions ouvertes. Ce taux modeste de réponse s'explique par le fait que seuls les rhumatologues pratiquant l'échographie musculosquelettique se sont sentis concernés par l'étude et ont donc participé à cette étude. Ceci ne compromet pas pour autant la valeur de cette enquête puisque notre objectif était de faire un état des lieux de l'échographie interventionnelle en France. 


\section{Conclusion}

L'échographie interventionnelle est de plus en plus pratiquée en France. Cet outil est devenu un complément essentiel à la pratique des rhumatologues permettant une prise en charge diagnostique et thérapeutique plus précoce et plus précise que la clinique seule. Le système de formation français de type universitaire est de bonne qualité mais le nombre de place reste encore insuffisant au vu de la demande qui ne cesse de croître ces dernières années. L'intégration de l'échographie musculo-squelettique au cours de la formation initiale du diplôme d'études spécialisé de rhumatologie doit être envisagée. Cette certification à l'échographie musculo-squelettique en général et à l'EMS interventionnelle en particulier devra être universitaire sous la forme d'un DU associé à d'autres formations pratiques organisées dans chaque inter-région sous la forme de séminaire de niveau croissant de compétences pouvant se dérouler sur un cycle de deux ans. La validation de chaque séminaire devra être obligatoire afin de passer au niveau supérieur et afin de pouvoir obtenir le diplôme d’étude spécialisé en rhumatologie.

\section{Déclaration d'intérêts}

Ce travail a été financé en partie par le Laboratoire Chugai. 


\section{Références}

[1] Kane D, Balint PV, Sturrock R, Grassi W. Musculoskeletal ultrasound--a state of the art review in rheumatology.Part 1: Current controversies and issues in the development of musculoskeletal ultrasound in rheumatology. Rheumatology (Oxford) 2004;43:823-8.

[2] Kane D, Grassi W, Sturrock R, Balint PV.Musculoskeletal ultrasound--a state of the art review in rheumatology.Part 2: Clinical indications for musculoskeletal ultrasound in rheumatology. Rheumatology (Oxford) 2004;43:829-38.

[3] Pineda C, Reginato AM, Flores V et al.Pan-American League of Associations for Rheumatology (PANLAR) recommendations and guidelines for muskuloskeletal ultrasound training in the Americas for rheumatologists. J Clin Rheumatol 2010;19:113-8.

[4] Larché MJ, Mc Donald-Blumer H, Bruns A et al.Utility and feasibility of musculoskeletal ultrasonography (MSK US) in rheumatology practice in Canada: needs assesment. Clin Rheumatol 2011;30:1277-83.

[5] Brown AK et al. Using ultrasonography to facilitate best practice in diagnosis and management of RA. Nat Rev Rheumatol 2009;5:698-706.

[6] Kane D, Balint PV, Sturrock RD. Ultrasonography is superior to clinical examination in the detection and localization of knee joint effusion in rheumatoid arthritis. J Rheumatol 2003;30:966-71.

[7] Schmidt WA. Value of sonography in diagnosis of rheumatoid arthritis. Lancet 2001;357:1056-7.

[8] Janow GL et al. Detection of active disease in juvenile idiopathic arthritis: sensitivity and specificity of th physical examination vs ultrasound. J Rheumatology 2011;38:2671-74.

[9] Ottaviani S, Bardin T, Richette P. Usefulness of ultrasonography for gout. Joint Bone Spine 2012;10.1016/j.jbspin.2012.01.012.

[10] Wakefield RJ, Green MJ, Marzo-Ortega H et al. Should oligoarthritis be reclassified? Ultrasound reveals a high prevalence of subclinical disease. Ann Rheum Dis 2004;63:382-5.

[11] Moore CL, Copel JA. Point-of-care ultrasonography. N Engl J Med 2011;364:749-57.

[12] Migliore A, Bizzi E, Massafra U et al. A new technical contribution for ultrasound-guided injections of sacro-iliac joints. Eur Rev Med Pharmacol Sci 2010;14:465-9.

[13] Cunnington J, Marshall N, Hide G et al. A randomized double-blind, controlled study of ultrasoundguided cortico-steroid injection into the joint of patients with inflammatory arthritis. Arthritis Rheum 2010;62:1862-9.

[14] Andrew Gilliland C, Salazar LD, Borchers JR. Ultrasound versus anatomic guidance for intra- articular and periarticular injection : a systematic review. Phys Sportsmed 2011;39:121-31.

[15] Hashiuchi T, Sakurai G, Sakamoto $Y$ et al. Comparative survey of pain-alleviating effects between ultrasound-guided injection and blind injection of lidocaine alone in patients with painful shoulder. Arch Orthop Trauma Surg 2010;130:847-52.

[16] Naredo E, Cabero F, Beneyto $P$ et al. A randomized study of short term response to blind injection versus sonographic-guided injection of local corticosteroids in patients with painful shoulder. J Rheumatol 2004;31:308-14. 
[17] Im SH, Lee SC, Park YB et al. Feasibility of sonography for intra-articular injections in the knee through a medial patellar portal. J Ultrasound Med 2009;28:1465-70.

[18] Khosla S, Thiele R, Baumhauer JF. Ultrasound guidance for intraarticular injections of the foot and ankle. Foot ankle Int 2009;30:886-90.

[19] Smith J, Finnoff JT, Levy BA, Lai JK. Sonographically guided proximal tibiofibular joint injection. J Ultrasound Med 2010;29:783-89.

[20] Raza K, Lee SC, Pilling D et al. Ultrasound guidance allows accurate needle placement and aspiration from small joints in patients with early inflammatory arthritis. Rheumatology (Oxford) 2003;42:976-79.

[21] Sibbitt WL Jr, Costantino TG, Filippone L, Satz W. Does sonographic needle guidance affect the clinical outcome of intrarticular injections? J Rheumatol 2009;36:1892-902.

[22] Mandl P, Naredo E et al. Practice of ultrasound -guided arthrocentesis and joint injection, including training and implementation, in Europe: results of a survey of experts and scientific societies. Rheumatology 2012;51:184-90.

[23] Naredo E, Bijlsma JWJ, Conaghan PG et al. Recommendations for the content and conduct of European League Against Rheumatisme (EULAR) musculoskeletal ultrasound courses. Ann Rheum Dis 2008;67:101722.

[24] Brown AK, O'Connor PJ, Wakefield RJ, et al. Practice, training, and assessment among experts performing musculoskeletal ultrasonography: toward the development of an international consensus of educational standards for ultrasonography for rheumatologists. Arthritis Rheum 2004;51:1018-22.

[25] Brown AK, O'Connor PJ, Roberts TE, et al. Recommendations for musculoskeletal ultrasonography by rheumatologists: setting global standards for best practice by expert consensus. Arthritis Rheum 2005;53:83-92.

[26] Brown AK, O'Connor PJ, Roberts TE, et al. Ultrasonography for rheumatologists : the development of specific competency based educational outcomes. Ann Rheum Dis 2006;65:629-36.

[27] Brown AK, Roberts TE, O'Connor PJ, et al. The development of an evidence-based educational framework to facilitate the training of competent rheumatologist ultrasonographers. Rheumatology (Oxford) 2007;46:391-97.

[28] Taggard A, Wright G, Ball E et al. The Belfast musculoskeletal ultrasound course. Rheumatology 2009;48:1073-76.

[29] Taggart A, Filippucci E, Wright G et al. Musculoskeletal ultrasound training in rheumatology: the Belfast experience. Rheumatology 2006;45:102-5.

[30] Cunnington J, Platt P, Raftery G, Kane D. Attitudes of United Kingdom rheumatologists to musculoskeletal ultrasound practice and training. Ann Rheum Dis 2007;66:1381-83.

[31] Tămaş MM, Fodor D, Rednic N, Rednic S. Musculoskeletal ultrasonography in Romania- Results from a specific questionnaire. Medical Ultrasonography 2011;1:10-4.

[32] Gaudin P. Ultrasonography and rheumatologist in private practice. La lettre du rhumatologue 2012;379-380:4-6. 
[33] Wakefield RJ, Goh E et al.Musculoskeletal ultrasonography in Europe: results of a rheumatologistbased survey at a Eular meeting. Rheumatology 2003;42:1251-3.

[34] Naredo E, Bijlsma JWJ. Becoming a musculoskeletal ultrasonographer. Best Pract Res Clin Rheumatol. 2009;23:257-67. 


\section{RAIVE - Enquête RhumAtologie InterVentionnelle et Échographie}

Vous êtes : $\square$ rhumatologue $\quad \square$ médecin du sport $\quad \square$ rééducateur

Année de thèse : I $1 \quad 1 \quad|\quad|$

Type d'activité : $\square$ libérale $\square$ hospitalière $\square$ mixte

Type de formation à l'échographie : $\square$ Echorhum $\quad \square$ ECRIN $\quad \square$ DU Regarde

$\square$ DU de radiologie $\quad \square$ autre, précisez :

Année de certification à l'échographie :

Avez-vous accès à un échographe?

$\square$ personnel

$\square$ dans le service où vous travaillez

$\square$ au cabinet de groupe

$\square$ chez le radiologue libéral en vacation

$\square$ dans le service de radiologie de l'hôpital où vous êtes vacataire

$\square$ pas du tout

Quelle est la référence de l'échographe que vous utilisez ?

Est-il : $\square$ loué $\square$ acheté $\square$ mis à disposition

Vous en changez tous les I__ ___ l ans

Nombre d'échographies interventionnelles/semaine : I________ échographies

En rhumatologie interventionnelle, vous utilisez : $\square$ la radiologie

$\square$ l'échographie
$\square$ I'un ou l'autre
$\square$ le guidage clinique uniquement

La pathologie majoritaire que vous explorez en échographie est : $\square$ rhumatismes inflammatoires

$\square$ traumatismes du sport et micro traumatologie tout venant

Travaillez-vous : $\quad \square$ seul $\quad \square$ aidé

Avez-vous un local dédié ? $\square$ oui $\square$ non 
Combien de gestes sous échographie pratiquez-vous par semaine? I___ I gestes

Avez-vous une plage horaire dédiée à ces gestes

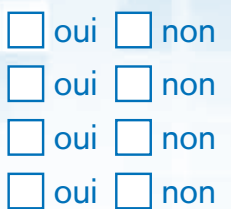

Les réalisez-vous au fil de la consultation?

Faites-vous une asepsie type HAS en 5 temps?

Utilisez-vous un autre produit que la bétadine?

oui $\square$ non

Si oui, lequel :

Quel est votre protocole?

Pour l'échoguidage, utilisez-vous du gel stérile?

Pour l'échoguidage, utilisez-vous une housse stérile pour la sonde?

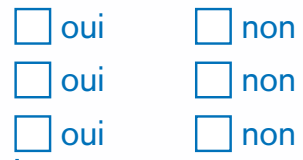

Avec votre protocole avez-vous eu des infections iatrogènes ?

Si oui, combien? I infections

Sur 100 gestes sous échographie, estimez le pourcentage que vous réalisez pour : - l'écho repérage I I (\%)

- l'écho guidage

Quelle est la raison de ce choix ? $\square$ rapidité $\square$ pas d'aide disponible $\square$ autre

Pour un geste sous échographie quel temps moyen y consacrez-vous ? I_ _ I I mn

Cotez-vous votre geste ? $\square$ oui $\square$ non

Quelle cotation utilisez-vous par exemple pour :

- une infiltration écho-guidée d'une bourse sous-acromiale (au cours d'une consultation avec examen clinique)

- l'évacuation d'un kyste poplité, écho-guidée, suivi d'une infiltration, sans écho du genou en intra-articulaire (au cours d' une consultation avec examen clinique)
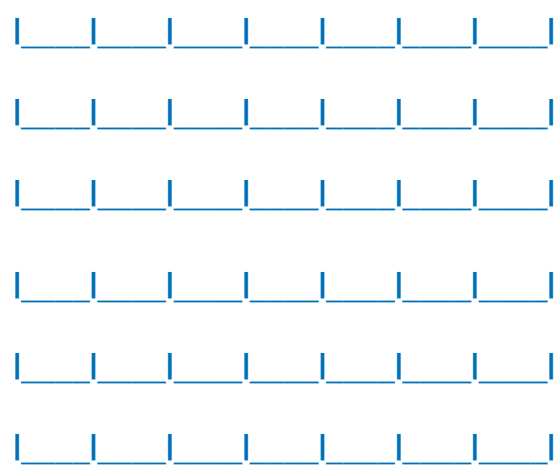

- l'évacuation et infiltration (dans le même temps), écho-guidée, d'un épanchement gléno-huméral

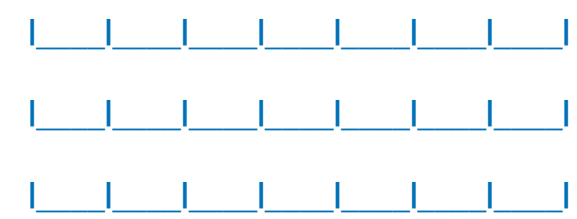

Cette activité de rhumatologie interventionnelle vous semble t-elle financièrement rentable ? $\square$ oui $\quad \square$ non

Souhaitez vous une formation ? $\square$ oui $\square$ non

Si oui : $\square$ à l'échographie seulement

$\square$ à l'échographie avec une application interventionnelle

$\square$ à la radiologie interventionnelle

Envisagez-vous une formation complémentaire ? 
THESE SOUTENUE PAR : Aurélie SICAUD

TITRE : RAIVE (RhumAtologie InterVentionelle et Echographie) : résultats d'une enquête nationale.

\section{CONCLUSION}

L'échographie interventionnelle est de plus en plus pratiquée en France. Cet outil est devenu un complément essentiel à la pratique des rhumatologues permettant une prise en charge diagnostique et thérapeutique plus précoce et plus précise que la clinique seule. Le système de formation français de type universitaire est de bonne qualité mais le nombre de places reste encore insuffisant au vu de la demande qui ne cesse de croître ces dernières années. L'intégration de l'échographie musculo-squelettique au cours de la formation initiale du diplôme d'études spécialisé de rhumatologie doit être envisagée. Cette certification à l'échographie musculo-squelettique en général et à l'EMS interventionnelle en particulier devra être universitaire sous la forme d'un DU associé à d'autres formations pratiques organisées dans chaque inter-région sous la forme de séminaire de niveau croissant de compétences pouvant se dérouler sur un cycle de deux ans. La validation de chaque séminaire devra être obligatoire afin de passer au niveau supérieur et afin de pouvoir obtenir le diplôme d'étude spécialisé en rhumatologie:

\section{VU ET PERMIS D'IMPRIMER}

\section{Grenoble, le $2019 / 2012$}

LE DOYEN

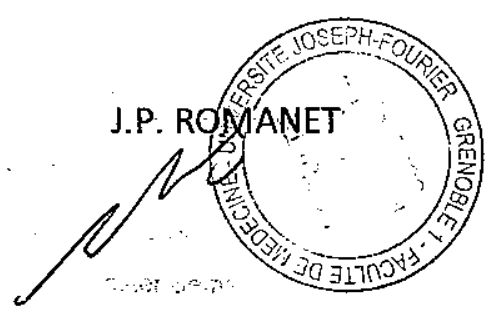

LE PRESIDENT DE LA THESE

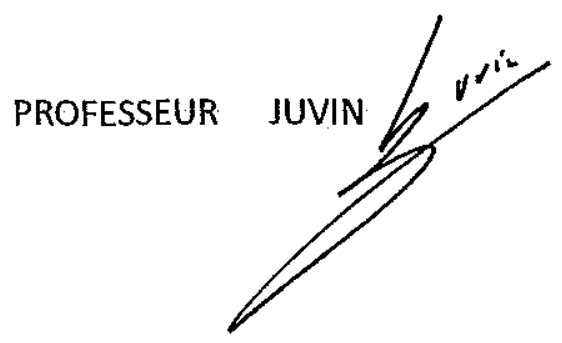




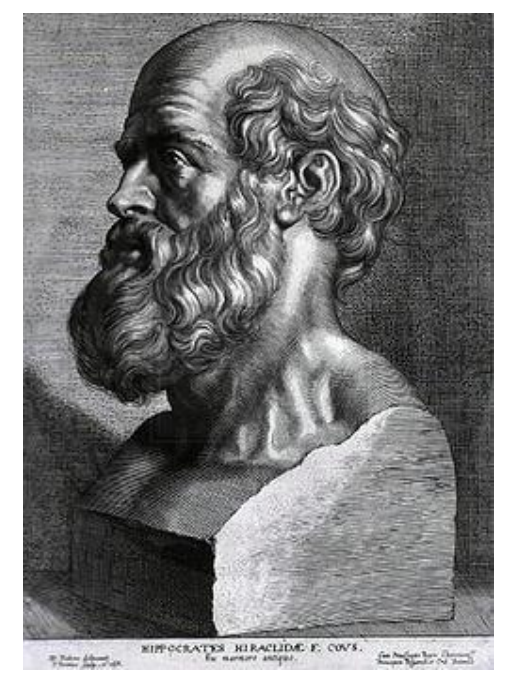

\section{SERMENT D'HIPPOCRATE}

En présence des Maîtres de cette Faculté, de mes chers condisciplines et devant I'effigie d'HIPPOCRATE,

Je promets et je jure d'être fidèle aux lois de l'honneur et de la probité dans l'exercice de la Médecine.

Je donnerais mes soins gratuitement à l'indigent et n'exigerai jamais un salaire au dessus de mon travail. Je ne participerai à aucun partage clandestin d'honoraires.

Admis dans l'intimité des maisons, mes yeux n'y verront pas ce qui s'y passe ; ma langue taira les secrets qui me seront confiés et mon état ne servira pas à corrompre les mœurs, ni à favoriser le crime.

Je ne permettrai pas que des considérations de religion, de nation, de race, de parti ou de classe sociale viennent s'interposer entre mon devoir et mon patient.

Je garderai le respect absolu de la vie humaine.

Même sous la menace, je n'admettrai pas de faire usage de mes connaissances médicales contre les lois de l'humanité.

Respectueux et reconnaissant envers mes Maîtres, je rendrai à leurs enfants l'instruction que j'ai reçue de leurs pères.

Que les hommes m'accordent leur estime si je suis fidèle à mes promesses.

Que je sois couvert d'opprobre et méprisé de mes confrères si j'y manque. 
